\title{
Improving the performance of three-mirror imaging systems with Freeform Optics
}

\author{
Joseph M. Howard and Steven Wolbach \\ NASA Goddard Space Flight Center, Johns Hopkins University \\ Joseph.M.Howard@nasa.gov,Swolbacl@jhu.edu.
}

\begin{abstract}
The image quality improvement for three-mirror systems by Freeform Optics is surveyed over various f-number and field specifications. Starting with the Korsch solution, we increase the surface shape degrees of freedom and record the improvements.

OCIS codes: (080.0080) Geometrical Optics; (080.4035) Mirror System Design; (080.4228) Nonspherical Mirror Surfaces
\end{abstract}

\section{Specifications and Starting Designs}

The performance of unobstructed three-mirror systems with varying surface definitions was investigated in this survey, with the goal of quantifying the improvement that freeform surfaces can offer. Each system was fixed with the object at infinity, and a $100 \mathrm{~mm}$ diameter circular entrance pupil was chosen to set the scale. The systems were all constrained to be symmetric about the $Y-Z$ plane. Four f-numbers were surveyed: $f / 5, f / 7.5, f / 10$, and f/20. Three field specifications were surveyed: 1,4 , and 5 degree half-field of view (HFOV) orthogonal of the plane of symmetry (i.e. along $\mathrm{X}$ ). A flat image surface was desired, and the field aspect ratio $(\mathrm{X} / \mathrm{Y})$ was fixed at 2.0, giving a rectangular format image surface, where the $\mathrm{Y}$ fields are half that of $\mathrm{X}$. Finally, systems both with and without an internal image were considered for each of the twelve combinations of field size and f-number, yielding 24 specific systems. For ease of differentiation here, systems with an internal image are referred to here as a TMA (threemirror-anastigmat), and systems without are referred to as an RT (reflective triplet).

Starting designs for each specific system were obtained using the freely available OSLO EDU [1], installed with the SLIDERS add-on macro feature [2]. This design tool contains a three-mirror telescope feature based on Korsch's closed form solution [3], enabling automated design of rotationally symmetric reflective systems composed of conic mirrors by simple manipulation of the slider feature in the software. A design for each of the 24 specific systems was chosen with similar configurations to that shown in Figure 1, where a TMA system (having an internal image) is laid out for the $\mathrm{f} / 10$ and 1 degree HFOV (in X, or out of the plane of the page). The independent parameters for each of the 24 systems consist of the primary mirror f-number, and the three separations (or thicknesses) between the mirrors and to the image surface. These parameter values were then used to transfer the models directly to CODE V [4], using a custom macro to set up the models for further optimization for this study.

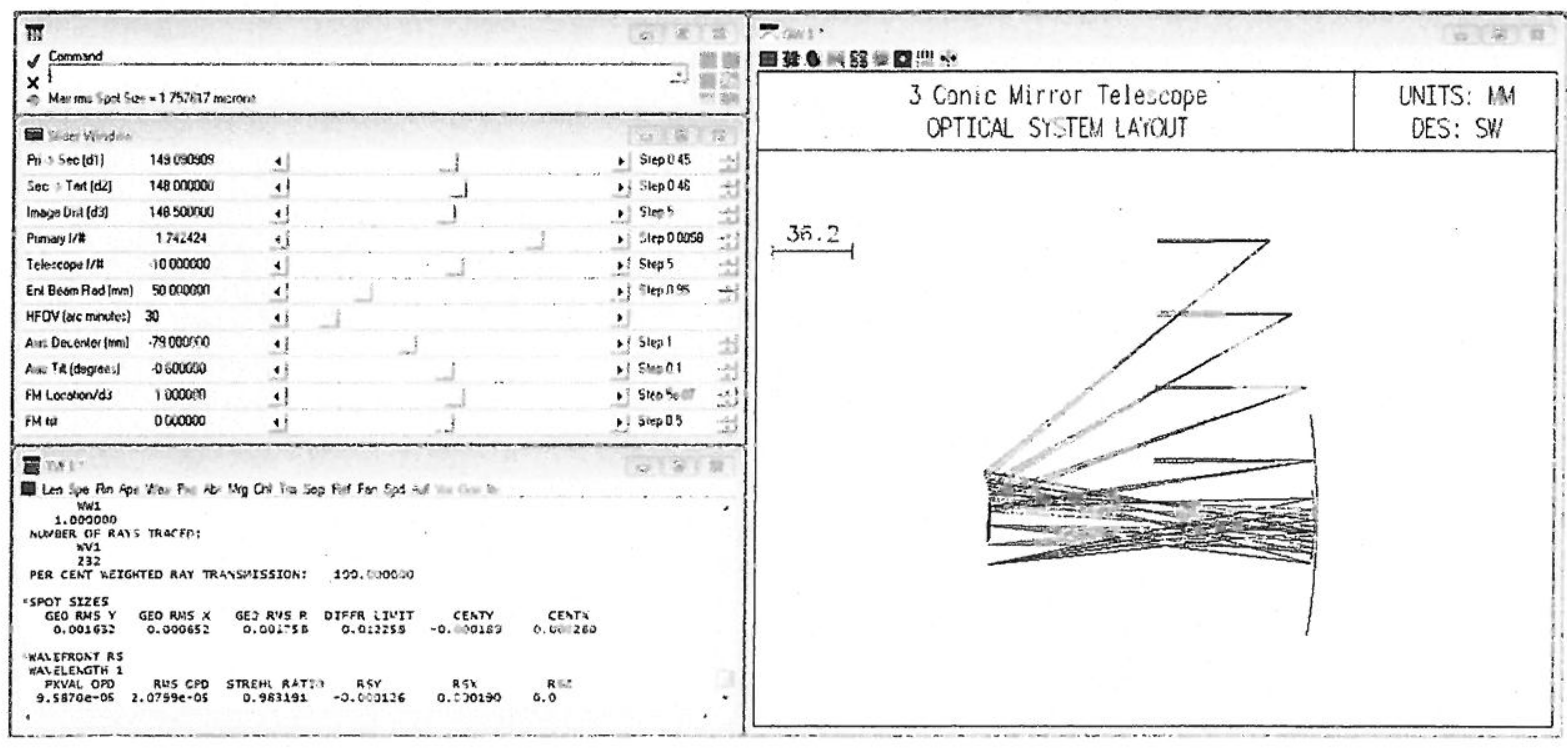

Figure 1. Screen-capture of OSLO EDU SLIDERS used in creating starting designs. 


\section{System performance improvement}

Each of the 24 imported systems were then locally optimized using the three conic constants and image tilt as variables to put them into the local numerical minimum with the default figure of merit in CODE V. To quantify the improvement, we evaluated the geometric RMS spot size over the field of view, and report the maximum value as a single performance metric for that system.

The systems were then improved by increasing surface shape degrees of freedom by both holding the optics stationary (i.e. minimizing package variations), and allowing for small movements via tilt and thickness variations, while always constraining the optimization against self obstruction. The progression of the surface shapes towards greater freeform shapes from the base conic form was: conic $\rightarrow$ conic with asphere $\left(6^{\text {th }}\right.$ and $8^{\text {th }}$ order $) \rightarrow$ biconic $\rightarrow$ Zernike surface (allowing the first thirteen "standard" terms to vary). All optimizations started with the "import" models imported from OSLO EDU, however the improvement factor reported in the next section is with respect to the locally optimized "import" model with the conic constants used as variables.

\section{Results}

As expected, each of the systems improved when more degrees of freedom were introduced into to the design. This improvement is quantified here as the ratio of the maximum root mean square (RMS) spot diameter before optimizing with the new degrees of freedom, to the RMS value afterwards. For example, the maximum RMS spot diameter over the $\mathrm{f} / 5$ and 5 -degree RT system before and after adding aspheres is 41.5 and 11.9 microns, respectively, so the improvement factor is simply $41.5 / 11.9=3.5$. Given this metric, all of the data can be plotted to illustrate the impact of freeform surfaces on each of the optical designs. Figure 2 shows the results for the RT systems for both optimization approaches (i.e. surface shape only, and then including tilts and thicknesses) using the maximum RMS spot radius over the field of view metric. By definition, the conic portion of both bar graphs are all set to unity since we are normalizing off of this starting point to evaluate the improvements by the introduction of freeform. As can be seen in the plot, the addition of aspheres yields improvements upwards to a factor of 10 for the surface shape only optimization, but only up to a factor of 5 when we break out of the original configuration. Using biconic surface profiles yield marginal improvements, and in some case worse results for this particular figure of merit (likely due to CODE V emphasizing the mean performance during optimization instead of the worst performance reported here). Zernike shaped surfaces, on the other hand, show considerable promise for this class of system.
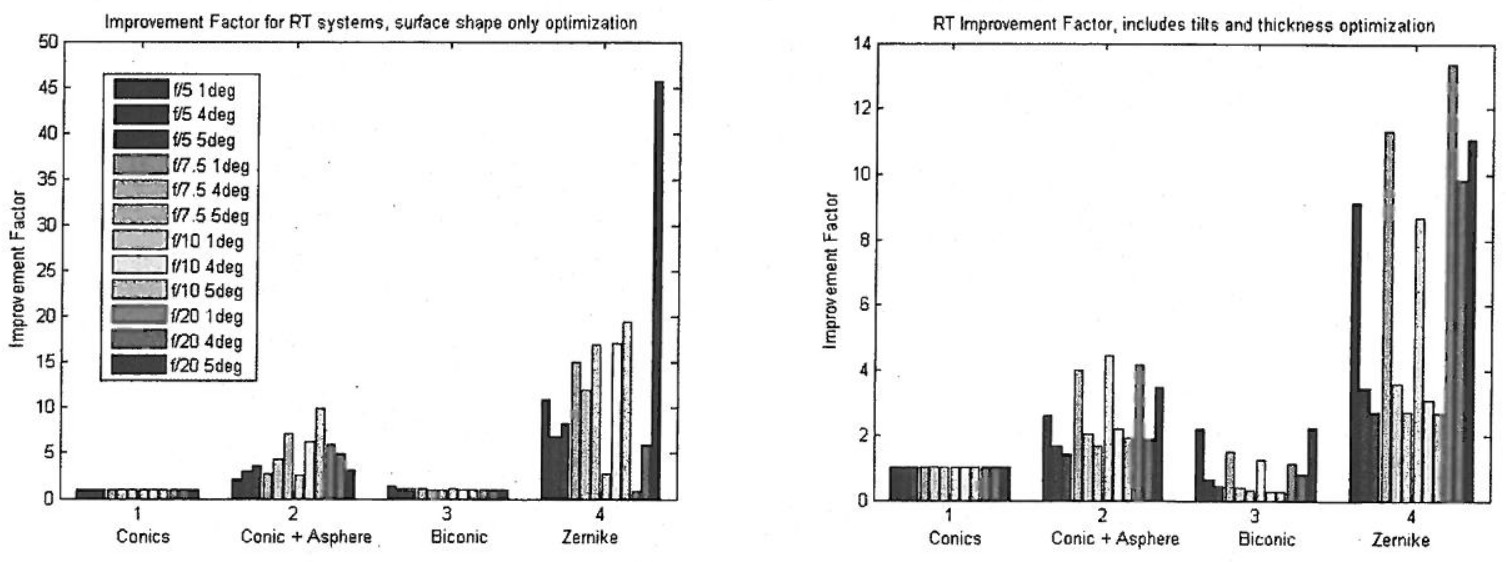

Figure 2. Improvements to RT class systems by adding greater surface shape degrees of freedom.

Results for TMA class systems are illustrated in Figure 3, where again the metric is the improvement factor of the maximum RMS spot diameter over the field of view. Here we see that the addition of aspheres has a less pronounced impact on system improvement, as does biconic surfaces, but once again Zernike surfaces show considerable improvements, upwards of a factor of 30 . 

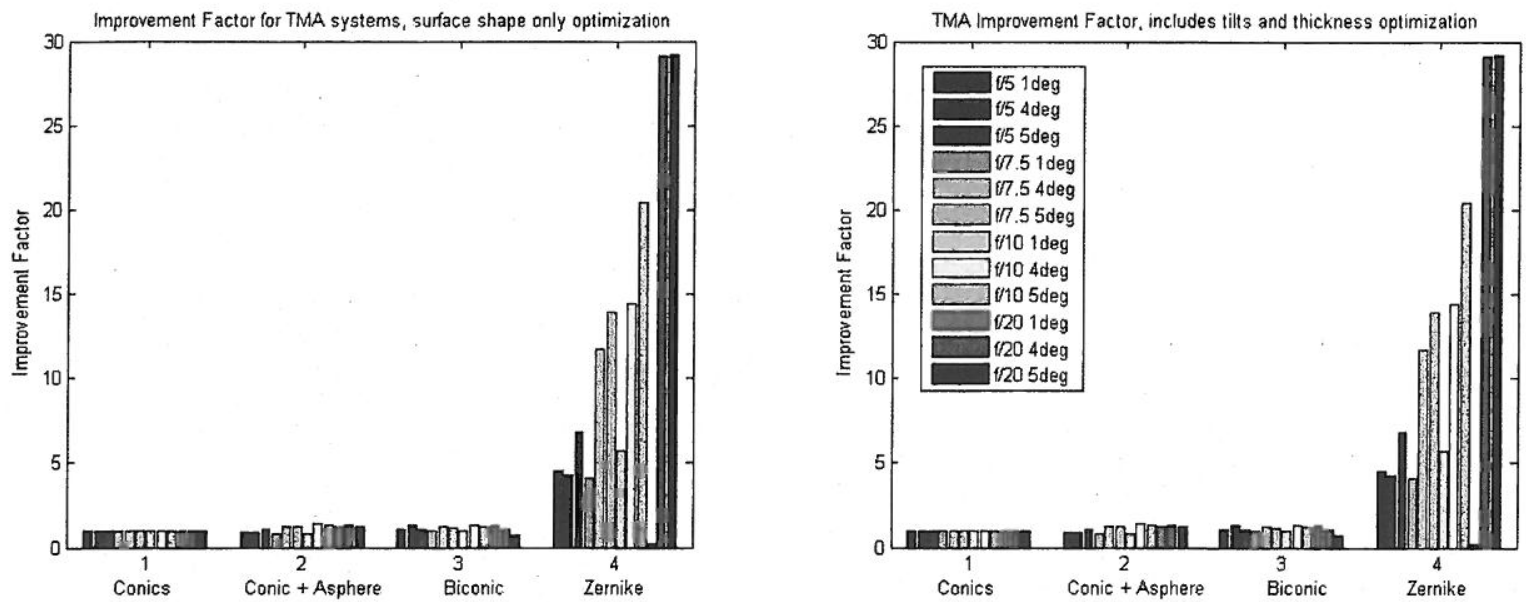

Figure 3. Improvements to TMA class systems by adding greater surface shape degrees of freedom.

\section{Concluding Remarks}

One of the primary motivations of this study was to quantify the potential improvement to optical systems that freeform surfaces can offer. This might help motivate their consideration and use by optical designers and their customers that can benefit from them. It is clear from Figures 2 and 3 that freeform surfaces such as Zernike profiles have enormous potential for improving optical systems. This finding is consistent with recently reported design improvements of a specific three-mirror reflective system [5]. Naturally, other surface shapes could have been considered in this study, including XY polynomials, splines, anamorphic aspheres, and Q-polynomials. These shapes (and others) could be directly compared to the performance improvement offered by Zernikes, ultimately leading to the development of the "best" surface shape definition for freeform optics for imaging systems.

Although not presented here, a "mix" of surface shapes was also briefly investigated to find the optimal impact on the design form. While all mirrors had the freeform shapes available for optimization in the data presented above, it turns out that some mirrors have greater impact than others for particular surface shapes (as one would expect), and that for a particular system, applying a freeform to a single surface can potentially yield the desired improvement. We also found that for some examples, a mix of biconics and Zernike surfaces yielded the best results.

\section{References}

[1] OSLO is a registered trademark of Lambda Research Corporation, http://www.lambdares.com.

[2] Joseph M. Howard, "SLIDERS: the next generation of automated optical design tools has arrived", Proc. SPIE 5174, 19 (2003).

[3] Dietrich Korsch, Reflective Optics (Academic Press, Boston, 1991).

[4] CODE V is a registered trademark of the Optical Solutions Group at Synopsys, http://optics.synopsys.com.

[5] Kyle H. Fuerschbach, Kevin P. Thompson and Jannick P. Rolland, "A new generation of optical systems with $\varphi$-polynomial surfaces", Proc. SPIE 7652, International Optical Design Conference 2010, 76520C (September 02, 2010). 